\title{
Dynamics of Unitarization by Classicalization
}

\author{
Gia Dvali ${ }^{a, b, c, d}$ and David Pirtskhalava ${ }^{d}$, \\ ${ }^{a}$ Arnold Sommerfeld Center for Theoretical Physics, Fakultät für Physik \\ Ludwig-Maximilians-Universität München, Theresienstr. 37, 80333 München, Germany \\ ${ }^{b}$ Max-Planck-Institut für Physik, Föhringer Ring 6, 80805 München, Germany \\ ${ }^{c}$ CERN, Theory Division, 1211 Geneva 23, Switzerland \\ ${ }^{d}$ CCPP, Department of Physics, New York University \\ 4 Washington Place, New York, NY 10003, USA
}

\begin{abstract}
We study dynamics of the classicalization phenomenon suggested in [1], according to which a class of non-renormalizable theories self-unitarizes at very high-energies via creation of classical configurations (classicalons). We study this phenomenon in an explicit model of derivatively-self-coupled scalar that serves as a prototype for a Nambu-Goldstone-Stückelberg field. We prepare the initial state in form of a collapsing wave-packet of a small occupation number but of very high energy, and observe that the classical configuration indeed develops. Our results confirm the previous estimates, showing that because of self-sourcing the wave-packet forms a classicalon configuration with radius that increases with center of mass energy. Thus, classicalization takes place before the waves get any chance of probing shortdistances. The self-sourcing by energy is the crucial point, which makes classicalization phenomenon different from the ordinary dispersion of the wave-packets in other interacting theories. Thanks to this, unlike solitons or other non-perturbative objects, the production of classicalons is not only unsuppressed, but in fact dominates the high-energy scattering. In order to make the difference between classicalizing and non-classicalizing theories clear, we use a language in which the scattering cross section in a generic theory can be universally understood as a geometric cross section set by a classical radius down to which waves can propagate freely, before being scattered. We then show, that in non-classicalizing examples this radius shrinks with increasing energy and becomes microscopic, whereas in classicalizing theories expands and becomes macroscopic. We study analogous scattering in a Galileon system and discover that classicalization is less efficient there. We thus observe, that classicalization is source-sensitive and that Goldstones pass the first test.
\end{abstract}




\section{Essence of Classicalization}

In the standard (Wilsonian) approach to UV-completion of quantum field theories, it is assumed, that non-renormalizable theories with cutoff $M_{*} \equiv L_{*}^{-1}$ necessarily violate unitarity above the scale $M_{*}$, and require its restoration by integratingin some external weakly-coupled degrees of freedom. This approach is based on a common physical intuition, that in a scattering process at higher and higher energies, $\sqrt{s} \gg M_{*}$, one is able to probe shorter and shorter distances, $L \sim 1 / \sqrt{s} \ll L_{*}$.

It has been suggested recently [1], that deep-UV picture can be very different in theories that contain extended classical objects (classicalons) that represent configurations of a bosonic field $\phi$ that is sourced by energy (momentum) of the scattering particles. The characteristic size of the classical configuration, called a classicalization radius $r_{*}$, is a model-dependent function of the energy of the source $\sqrt{s}$, but the key feature is, that for the trans-cutoff energies $\sqrt{s} \gg M_{*}$, the classicalization radius exceeds the fundamental length $r_{*}(\sqrt{s}) \gg L_{*}$. An essential condition for classicalization is the existence of a classicalizer field, $\phi$, which is sourced by the energy-momentum sources that are involved in a given scattering process. The role of the classicalon is to form an extended classical configuration of size $r_{*}(\sqrt{s})$, whenever the energy associated with a given process exceeds the cutoff scale $M_{*}$. In this way, classicalon produces a barrier for probing sub-cutoff distances in a given scattering process 1 .

The consequences of such a picture would be pretty profound. Because of classicalization, it becomes impossible to localize energy at distances shorter than the corresponding $r_{*}$-radius. As a result, the $2 \rightarrow 2$ particle scatterings at large center of mass energy $\sqrt{s} \gg M_{*}$ happen only through very low momentum-transfer $\sim r_{*}^{-1}$, and give negligible contribution to the scattering process. Instead, the cross section is dominated by creation of classical configurations that decay into many particle states.

From the first glance, such a scenario may look puzzling, since creation of classical objects, such as topological solitons, in two-particle collisions must be exponentiallysuppressed. So why are the classicalons created? The answer is, because classicalons are not solitons. They are configurations of the $\phi$-field sourced by the energy, which is a Noether-type rather than a topological-type charge. Therefore, appearance of a classicalon configuration is inevitable whenever localization of high-enough energy takes place. Correspondingly, in processes that exhibit classicalization, it is simply impossible to form two-particle quantum states with energy $\sqrt{s} \gg M_{*}$, localized at distances $\ll r_{*}(\sqrt{s})$ ! Any such localized state is automatically a classical object and thus, represents a many-particle state. In other words, when preparing the scattering experiment by bringing two highly energetic particles within the impact parameter from an initially-infinite separation, we are gradually building a classical

\footnotetext{
${ }^{1}$ As pointed out in [1] $r_{*}$-radius plays the role somewhat analogous to Schwarzschild radius for non-gravitational theories, whereas classicalons share some obvious analogy with black holes, in the sense that they both represent strong sources of the corresponding classicalon fields.
} 
configuration via sourcing the classicalizer field by the center of mass energy of the colliding particles. By the time particles come within the $r_{*}(\sqrt{s})$-radius corresponding to their center of mass energy, the system is already classical and no longer represents a two-particle quantum state. So effectively the only chance for $2 \rightarrow 2$ scattering to take place is, if particles never come closer than $r_{*}$-distance. Thus, in $2 \rightarrow 2$ particle scattering, the momentum transfer is limited by $r_{*}(\sqrt{s})^{-1}$.

In other words, classicalon configurations are a necessary consequence of localized energy, because the latter sources the classicalizer field, and system has no other choice, but to produce one. This goes in a sharp difference with solitons or other non-perturbative objects that represent very special coherent states among the exponentially-large number of possible states compatible with the same energy. This is why in two (or few) particle collisions production of the latter objects is exponentially-suppressed even at very high energies [2].

Obviously, there are many open questions, most pressing of which is, does classicalization really work?

In this note, following [1], we shall take a small step towards understanding the dynamics of classicalization. Our idea is to prepare an initial wave-packet with very high-energy $\sqrt{s} \gg M_{*}$ but a relatively small occupation number, and investigate how the classical configuration of radius $r_{*}(\sqrt{s}) \gg L_{*}$ develops in the scattering process.

In order to better understand physics behind the classicalization phenomenon, we shall first confront classicalizing theories with the ones that do not exhibit this phenomenon. For a better comparison we shall develop an universal language, in which the scattering cross section in a generic theory can be understood as a geometric cross-section defined by the radius $r_{*}$. The physical meaning of the $r_{*}$-radius is of a shortest distance down to which in a given scattering process waves can propagate freely, without experiencing a significant interaction. In this language, the scattering cross section is universally given by,

$$
\sigma \sim r_{*}(\sqrt{s})^{2}
$$

This relation holds true both in weakly-coupled examples that do not classicalize, as well as in classicalizing theories. For example, for Thomson scattering, the role of $r_{*}$ is played by the classical radius of electron.

However, what distinguishes the two cases is the dependence of $r_{*}$-radius on energy. In non-classicalizing theories $r_{*}(\sqrt{s})$ diminishes with growing $\sqrt{s}$, whereas in classicalizing theories it grows and eventually becomes macroscopic. As a result, in the former theories particles can probe shorter and shorter distances $(\sim 1 / \sqrt{s})$, before being scattered, whereas in classiclizing theories scattering takes place already at $r_{*}$.

The analogy between $r_{*}$ and the gravitational Schwarzschild radius is clear. We can define a classical Schwarzschild radius for an arbitrary quantum particle, but a particle for which the Schwarzschild radius is deep within its Compton wavelength 
cannot be regarded as a classical state. Similarly, the classical $r_{*}$-radius can be defined for an arbitrary wave-packet, but only the ones with $r_{*} \gg L_{*}$ classicalize.

We shall examine the two systems. The first is a theory of a derivatively selfinteracting scalar that serves as a prototype of the Nambu-Goldstone boson or equivalently of longitudinal (Stückelberg) component of a massive vector field. We study $S$-wave scattering and observe that the configuration indeed classicalizes due to self-sourcing of the wave-packet. Both, for monochromatic waves as well as for sharply-localized wave-packets, we find that the $r_{*}$-radius is given by,

$$
r_{*}(\sqrt{s})=L_{*}\left(L_{*} \sqrt{s}\right)^{\frac{1}{3}},
$$

which is in agreement with the estimates of [1]. Corresponding $2 \rightarrow 2$ scattering amplitude then is estimated as,

$$
A_{2 \rightarrow 2} \sim\left(L_{*} \sqrt{s}\right)^{-\frac{4}{3}}
$$

whereas the total cross-section (dominated by the production of classical configuration) is given by,

$$
\sigma \sim L_{*}^{2}\left(L_{*} \sqrt{s}\right)^{\frac{2}{3}}
$$

All the above indicates that Goldstone-type derivatively-coupled scalars are interesting candidates for classicalization. Of course, as explained in [1, for classicalization to work, it is essential that there exist no light (with mass $\ll M_{*}$ ) weakly-coupled radial degree of freedom, otherwise system never classicalizes, and instead, takes the conventional root of a weakly-coupled perturbative unitarization.

We next explore the situation for another scalar theory, which describes a certain decoupling limit of a generally-covariant theory of a massive resonance graviton (DGP gravity[3]). In this limit the only remaining interacting component of the graviton is a helicity-zero Stückelberg component of the massive spin-2 state. We discover that unlike the Goldstone case, in the latter theory in the $S$-wave scattering classicalization does not happen in the leading order in self-coupling. The reason can be traced to the Galilean symmetry [4] that requires an excessive number of derivatives in the self-coupling of the scalar.

We confront derivatively-(self)coupled bosons with other types of self-interacting scalars and show the crucial role of energy self-sourcing for classicalization. Since energy is conserved, the scalars that couple to energy density necessarily get a very strong source, whenever energy is localized, and classicalize. This does not hold if the source is not of the energy-type. Classicalization thus is different from usual dispersion of the wave-packet in self-interacting theories.

We thus observe, that classicalization is sensitive to the nature of self-sourcing, and that Nambu-Goldstone-type scalars pass the first test.

\section{Scattering with and without Classicalization}

We wish to give a preliminary discussion of the classicalization phenomenon in a scattering process. In order to understand the peculiarity of a classicalizing scat- 
tering, we wish to confront it with scattering in theories where classicalization does not happen.

In order to achieve a maximally clear comparison, it is useful to reduce the derivation of the cross section in both classes of theories to a common language, in which the cross section can be understood as geometric cross-section set by $r_{*}$-radius, a shortest distance till which waves can propagate freely, before being scattered by interaction. With this definition, the concept of $r_{*}$-radius extends to weakly-coupled non-classicalizing theories. This extension allows us an universal treatment.

We shall then see, that in non-classicalizing weakly-coupled theories this distance diminishes with energy, whereas in the classicalizing theories grows. This is the fundamental difference between the theories that classicalize from the ones that do not exhibit such a phenomenon.

\subsection{Understanding cross-section as geometric cross-section}

As the first example of non-classicalizing theory, we shall consider a theory of massless scalar field with a renormalizable self-interaction,

$$
\mathcal{L}=\frac{1}{2}\left(\partial_{\mu} \phi\right)^{2}-\frac{\lambda}{4} \phi^{4}
$$

At the weak coupling, $\lambda \ll 1$, the $2 \rightarrow 2$ perturbative scattering amplitude in the above theory goes as

$$
A_{2 \rightarrow 2} \sim \lambda
$$

and the cross section goes as

$$
\sigma_{2 \rightarrow 2} \sim \frac{\lambda^{2}}{s}
$$

In this theory a tree-level scattering can probe arbitrarily-short distances, and classicalization does not happen. In order to understand this fact, let us try to understand the behavior of the scattering amplitude and of the cross-section in the language of solving the equation of motion,

$$
\square \phi=-\lambda \phi^{3},
$$

for scattering wave-packets. We shall assume that for $r=\infty$ and $t=-\infty, \phi$ is well-approximated by a spherical wave of frequency $\omega$, and the amplitude $A \sim 1$ (small occupation number),

$$
\phi_{0}=\frac{\psi(\omega(r+t))}{r},
$$

which solves the free-field equation of motion,

$$
\square \phi_{0}=0 .
$$

We shall now solve the equation (8) iteratively, by expanding the $\phi$-field in series of sub-leading corrections,

$$
\phi=\phi_{0}+\phi_{1}+\ldots
$$


and trying to understand at what distances the correction $\phi_{1}$ to a free-wave becomes significant. For this we have to solve the equation,

$$
\square \phi_{1}=-\lambda \phi_{0}^{3}=-\lambda \frac{\psi(\omega(r+t))^{3}}{r^{3}} .
$$

The solution of this equation, for $\omega \gg r^{-1}$, behaves as,

$$
\phi_{1} \simeq-\frac{\lambda}{2} \frac{\int_{0}^{r+t} \psi(\omega y)^{3} d y}{r^{2}}
$$

Notice, that since $\psi(w y)$ is a periodic function with amplitude $\sim 1$ and frequency $\omega$, we have (on average),

$$
\int_{0}^{r+t} \psi(\omega y)^{3} d y \sim \frac{1}{\omega} \psi(w(r+t)) \sim \frac{1}{\omega} .
$$

For example, taking $\psi=\cos (\omega(t+r))$ we get,

$$
\phi_{1}=-\frac{\lambda}{6} \frac{\sin (\omega(t+r))\left(2+\cos ^{2}(\omega(t+r))\right)}{\omega r^{2}} .
$$

Thus we find,

$$
\phi_{1} \sim \frac{\lambda}{\omega r} \phi_{0}
$$

This relation plays the central role in deriving the scattering cross-section in $\phi^{4}$ theory, since it shows, that the wave propagates freely till the distance,

$$
r_{*} \sim \frac{\lambda}{\omega} .
$$

What is the significance of the above relation? It tells us, that the high-frequency modes go deeper in UV before being disturbed by the interaction. Thus, the scattering becomes less and less significant for larger and larger energies. However at $r \sim r_{*}$, the correction to the free-wave becomes order one, and the scattering takes place. The scattering cross section thus can be understood as a geometric cross section,

$$
\sigma \sim r_{*}^{2} \sim \frac{\lambda^{2}}{\omega^{2}}
$$

which reproduces (7). The above cross section diminishes at high energies because the energetic waves probe shorter and shorter distances. Let us confront the above situation with a theory in which the field is self-sourced by the energy. As we shall see, the outcome is very different there. 


\subsection{Classicalization in Goldstone Scattering}

The example now we wish to consider is of a scalar field $\phi$ with the derivative self-interactions,

$$
\mathcal{L}=\frac{1}{2}\left(\partial_{\mu} \phi\right)^{2}+\frac{L_{*}^{4}}{4}\left(\left(\partial_{\mu} \phi\right)^{2}\right)^{2}
$$

This theory is symmetric under the shift by an arbitrary constant $c$,

$$
\phi \rightarrow \phi+c,
$$

and therefore represents a simple prototype describing a self-interacting NambuGoldstone field $\phi$, or a longitudinal (Stückelberg) component of a massive vector field. As shown in [5], the above theory can be regarded as the decoupling limit of the self-interacting theory of a massive Proca vector field $W_{\mu} \equiv \tilde{W}_{\mu}-m_{W}^{-1} \partial_{\mu} \phi$, with the following Lagrangian

$$
\mathcal{L}=-\frac{1}{4} F_{\mu \nu} F^{\mu \nu}+\frac{1}{2} m_{W}^{2} W_{\mu} W^{\mu}+\frac{g^{4}}{4}\left(W_{\mu} W^{\mu}\right)^{2} .
$$

The Lagrangian (19) is obtained from (21) in the limit $g \rightarrow 0$ with $L_{*} \equiv g / M_{W}=$ fixed. In this limit, the transverse gauge field decouples and we are left with a selfinteracting Nambu-Goldstone-Stückelberg mode, $\phi$. This theory is known to classicalize for the localized external probe sources. We wish to study classicalization in a simplest scattering process.

Perturbatively, at the frequency $\omega$, the amplitude of the $2 \rightarrow 2$ scattering process in the above theory goes as $A_{2 \rightarrow 2} \sim \omega^{4} L_{*}^{4}$, and naive perturbative cross section is dominated by the high-momentum transfer processes, growing as

$$
\sigma_{2 \rightarrow 2} \sim L_{*}^{8} \omega^{6}
$$

thus, violating unitarity for $\omega \gg M_{*}$. We now wish to check if this conclusion holds for non-perturbative analysis.

For this we shall study the scattering of waves by directly analyzing the equation of motion following from (19), which reads as follows

$$
\partial^{\mu}\left(\partial_{\mu} \phi\left(1+L_{*}^{4}\left(\partial_{\nu} \phi\right)^{2}\right)\right)=0
$$

As in the case of analyzing scattering in $\phi^{4}$-theory, we shall assume that for $r=\infty$ and $t=-\infty, \phi$ is well-approximated by a spherical wave of frequency $\omega$, and the amplitude $A \sim 1$ given by (9), and solve the equation iteratively by performing the expansion (11). The equation for the leading correction to the free wave now becomes,

$$
\square \phi_{1}=-L_{*}^{4} \partial^{\mu}\left(\partial_{\mu} \phi_{0}\left(\partial_{\nu} \phi_{0}\right)^{2}\right) \text {. }
$$

Taking into the account properties of $\psi(\omega(t+r))$-wave, for $\omega \gg r$, the leading contribution to the right hand side is,

$$
\square \phi_{1}=-\frac{L_{*}^{4}}{r^{5}}\left(2 \psi^{2} \psi^{\prime \prime}+8 \psi \psi^{\prime 2}\right),
$$


where prime denotes the derivative with respect to the argument. Again, for $\omega \gg$ $r^{-1}$ the solution of this equation can be approximated by,

$$
\phi_{1} \simeq-f(\omega(r+t)) \frac{L_{*}^{4}}{6 r^{4}}
$$

where,

$$
f(\omega(r+t)) \equiv \int_{0}^{r+t}\left(2 \psi^{2} \psi^{\prime \prime}+8 \psi \psi^{\prime 2}\right) d y .
$$

Notice, that since $\psi(w y)$ is a periodic function of amplitude $\sim 1$ and frequency $\omega$, we have,

$$
f \sim \omega \psi \sim \omega
$$

This relation can be readily checked on an explicit form of $\psi$. For example, for $\psi=\cos (\omega(t+r))$ we have, $f=\omega(3 \sin (\omega(t+r))-5 \sin (3 \omega(t+r))) / 6$.

Thus, we obtain the following relation between the initial free-wave and the leading perturbation due to scattering,

$$
\phi_{1} \sim\left(\frac{r_{*}}{r}\right)^{3} \phi_{0}
$$

where,

$$
r_{*} \equiv L_{*}\left(\omega L_{*}\right)^{\frac{1}{3}} .
$$

This equation tells us, that unlike the $\phi^{4}$-case, the scattering of Goldstones starts already at a distance $r_{*}$, which grows at large $\omega$. For $\omega \gg L_{*}$, the $2 \rightarrow 2$ particle scattering is thus dominated by a very low momentum transfer $\sim r_{*}^{-1}$, and must go as

$$
A_{2 \rightarrow 2} \sim\left(L_{*} / r_{*}\right)^{4} .
$$

Thus, scattering softens at high energy. This is a consequence of classicalization. Because of energy self-sourcing, the initial wave adiabatically re-scatters and by the time it reaches $r_{*}$, the correction to a free-wave becomes important. High energy scattering becomes governed by a long-distance physics.

\section{Classicalization of Localized Goldstone Wave- Packets}

We have seen, that scattering of monochromatic waves leads to the formation of $r_{*}$-radius, which indicates, that with growing energy, waves tend to scatter before reaching short distances. Thus two-to-two particle scatterings must be dominated by very low momentum-transfer. The high momentum-transfer scattering on the other hand should be accompanied by formation of a configuration of classical radius $r_{*}$. The system classicalizes at high $\sqrt{s}$. We wish to show that this property persists for the sharply localized wave-packets. 
Thus, we shall now consider classicalization of sharply-localized wave-packets. We shall limit our analysis to studying classicalization in $S$-wave scattering. We wish to see if and how the energetic wave-packets "refuse" to get localized and develop the $r_{*}$-radius. For this, we shall prepare the initial state of $\phi$ in the form of a collapsing spherical wave-packet of radius $r$, amplitude $A$, and a characteristic thickness $a$,

$$
\phi_{0}=\frac{\psi((r+t) / a)}{r}
$$

where $\psi(x / a)$ represents a sharply localized function of width $a$ around $x=0$. For small $A$ and small $a$ the initial wave-packet can be considered as a quantum state with low occupation number, which however may have an arbitrarily high energy, $\sqrt{s} \sim A^{2} / a$.

The spherical wave-packet starts at $t=-\infty$ at $r=\infty$, and collapses towards the origin. We shall study its spread-out in the course of this evolution. For this we shall solve the equation (23) iteratively, by building up perturbations in powers of $L_{*}^{4}$ around the solution $\phi_{0}$ of the linearized equation,

$$
\phi=\phi_{0}+\phi_{1}+\ldots
$$

As we shall see, as long as $a<r, \phi_{1} \sim \frac{A^{3} L_{*}^{4}}{a r^{4}}$ and the role of a small expansion parameter is therefore played by the following quantity

$$
\epsilon \equiv A^{2} L_{*}^{4} /\left(a r^{3}\right)
$$

Thus, the first order-in- $\epsilon$ correction to the free, collapsing wave-packet can be found as the solution of the following equation,

$$
\square \phi_{1}=-L_{*}^{4} \partial^{\mu}\left(\partial_{\mu} \phi_{0}\left(\partial_{\nu} \phi_{0}\right)^{2}\right),
$$

where the $\phi_{0}$-dependent part acts as a source for $\phi_{1}$.

We shall solve this equation in two different ways.

First, in order to develop an intuition, we shall replace the source by a timedependent point-like source of the strength that at each $t$ equals to a spatiallyintegrated value of the exact source.

Later, we shall solve the equation exactly without the former approximation and observe that to the leading order the two procedures give the solutions with identical asymptotic behavior, up to a multiplicative factor $-4 / 3$, which is consistent with the averaging procedure.

\subsection{Classicalization from the averaged source}

We shall discuss the method of the average source first. Since for any given $t, \phi_{0}$ is localized within the radius $r \sim|t|$, we can integrate the source over a sphere of 
radius $\gg|t|$ and at distances $r \gg|t|$ use an effective equation with a delta-function type source,

$$
\square \phi_{1}=\delta(\vec{r}) Q(t)
$$

Here

$$
Q(t)=-L_{*}^{4} \int_{R \gg|t|} d^{3} x \partial^{\mu}\left(\partial_{\mu} \phi_{0}\left(\partial_{\nu} \phi_{0}\right)^{2}\right)
$$

where volume integration is performed within a sphere of radius $R \gg|t|$. Since $\phi_{0}$ is a localized function, the integral of the spatial divergence

$$
\int_{R \gg|t|} d^{3} x \partial^{j}\left(\partial_{j} \phi_{0}\left(\partial_{\nu} \phi_{0}\right)^{2}\right)=0
$$

is the surface flux, which vanishes through any sphere outside the wave-packet. Thus, we are left with the following effective source,

$$
Q(t)=-L_{*}^{4} \int d^{3} x \partial_{t}\left(\partial_{t} \phi_{0}\left(\partial_{\nu} \phi_{0}\right)^{2}\right)
$$

Taking into the account the form of the zeroth order solution (32), we get,

$$
Q(t)=4 \pi L_{*}^{4} \int d r\left(\frac{\left(\psi^{\prime} \psi^{2}\right)^{\prime}}{r^{3}}-\frac{\left(2 \psi \psi^{\prime 2}\right)^{\prime}}{r^{2}}\right)
$$

where prime represents a derivative with respect to $r+t$ (which is the same as $\partial_{r}$ or $\left.\partial_{t}\right)$. After a few partial integrations, we obtain,

$$
Q(t)=16 \pi L_{*}^{4} \int d r\left(\frac{\psi^{3}}{r^{5}}-\frac{\left(\psi \psi^{\prime 2}\right)}{r^{3}}\right) .
$$

Let us now assume a particular form of the zeroth-order solution,

$$
\phi_{0}=\frac{\psi(r+t)}{r}=A \frac{e^{-\frac{(r+t)^{2}}{a^{2}}}}{r}
$$

which represents a wave-packet of amplitude $A$ and energy $\sim A^{2} / a$. Using this expression, we can evaluate the source in (40)

$$
Q(t)=-16 \pi L_{*}^{4} A^{3} \int d r\left(\frac{2}{3} \frac{1}{a^{2} r^{3}}+\frac{1}{9 r^{3}} \partial_{r}^{2}-\frac{1}{r^{5}}\right) e^{-\frac{3(r+t)^{2}}{a^{2}}} .
$$

We will now take the limit of an infinitely-strongly localized wave-packet $a \rightarrow 0$, so that the last expression becomes

$$
Q(t)=-16 \pi L_{*}^{4} A^{3} \sqrt{\frac{\pi}{3}} \int d r\left(\frac{2}{3} \frac{1}{a r^{3}}+\frac{a}{9 r^{3}} \partial_{r}^{2}-\frac{a}{r^{5}}\right) \delta(r+t) .
$$


The first term on the right hand side of the latter equation, being enhanced by inverse of the wave-packet width, represents the leading contribution to the effective source for $|t| \gg a$,

$$
Q(t \ll-a)=\frac{32 \pi}{3} \sqrt{\frac{\pi}{3}} L_{*}^{4} A^{3} \frac{1}{a t^{3}} .
$$

The solution of (35) with the above source gives the spread of the wave-packet for $r \gg|t|$

$$
\phi_{1}=\frac{Q(t-r)}{4 \pi r}=\frac{8}{3} \sqrt{\frac{\pi}{3}} L_{*}^{4} A^{3} \frac{1}{a(t-r)^{3} r} .
$$

This solution indicates that an energetic wave-packet of $\phi$ (with $\sqrt{s} \sim A^{2} / a \gg M_{*}$ ) classicalizes, since the value of the gradient reaches $M_{*}^{2}$ at distances $r_{*} \gg L_{*}$. We shall come back to a more precise estimate of $r_{*}$ shortly.

As a next step, we shall obtain the exact solution of (34), without averaging the source.

\subsection{Exact solution}

Below we will solve the equation (34) explicitly, by taking the same initial-state wave-packet $\phi_{0}$ as before, and constructing the perturbative solution. The details of derivation of the perturbative solution are given in the appendix. Here we shall reproduce only the essential steps.

Let us introduce the exact source $j(r, t)$, defined by

$$
j(r, t) \equiv \square \phi_{1}=-L_{*}^{4} \partial^{\mu}\left(\partial_{\mu} \phi_{0}\left(\partial_{\nu} \phi_{0}\right)^{2}\right) .
$$

Taking (32) into the account, the source can be rewritten as,

$$
j(r, t)=-L_{*}^{4}\left(2 \frac{\psi^{2} \psi^{\prime \prime}}{r^{5}}+8 \frac{\psi \psi^{2}}{r^{5}}-12 \frac{\psi^{2} \psi^{\prime}}{r^{6}}+4 \frac{\psi^{3}}{r^{7}}\right) .
$$

Evaluating the latter expression for the zeroth order wave-packet $\psi=A e^{-\frac{(r+t)^{2}}{a^{2}}}$, and later taking the $a \rightarrow 0$ limit as above, $j(r, t)$ can be recast in the following form

$$
j(r, t)=-L_{*}^{4} A^{3} \sqrt{\frac{\pi}{3}}\left(\frac{8}{3 a r^{5}}-\frac{4 a}{r^{6}} \partial_{r}+\frac{10 a}{9 r^{5}} \partial_{r}^{2}+\frac{4 a}{r^{7}}\right) \delta(r+t) .
$$

The equation for the perturbation (46) is then explicitly solved by (see the appendix),

$\phi_{1}=-\frac{4}{9} L_{*}^{4} A^{3} \sqrt{\frac{\pi}{3}}\left(\frac{8}{a} \frac{\theta(r+t)}{(t-r)^{3} r}+\frac{10 a}{3} \frac{\partial_{t} \delta(r+t)}{(t-r)^{3} r}-2 a \frac{\delta(r+t)}{(t-r)^{4} r}-\frac{16 a}{5} \frac{\theta(r+t)}{(t-r)^{5} r}\right)$,

which for $r>>a$, reduces to

$$
\phi_{1}(r \gg a)=-\frac{32}{9} \sqrt{\frac{\pi}{3}} L_{*}^{4} A^{3} \frac{\theta(r+t)}{a(t-r)^{3} r} .
$$


Notice, that the latter expression includes an extra factor of $-4 / 3$ as compared to the solution (45) obtained by the averaging of the source. This is not surprising, given the fact that the collapsing pulse moves at the speed of light, and the approximation of an instant averaging should give an order-one correction 2 .

The solution (50) shows that a collapsing spherical wave leaves a wake of radial "electric" field $\partial_{j} \phi$ and therefore classicalizes.

\section{Estimate of $r_{*}$-radius}

We shall now estimate the $r_{*}$-radius of the classicalon. The most straightforward estimate comes from the condition that for $r \sim r_{*}$, the expansion parameter $\epsilon$, defined in (33), becomes order one,

$$
\epsilon_{*} \equiv A^{2} L_{*}^{4} /\left(a r_{*}^{3}\right) \sim 1
$$

which gives,

$$
r_{*} \sim L_{*}\left(A^{2} L_{*} / a\right)^{1 / 3} .
$$

Translating this expression in terms of the center of mass energy

$$
\sqrt{s} \sim \frac{A^{2}}{a}
$$

we get

$$
r_{*}(s) \sim L_{*}\left(L_{*} \sqrt{s}\right)^{\frac{1}{3}},
$$

which reproduces the estimate of [1].

The above estimate agrees with the following one: the radius $r_{*}$ is defined as the maximal value of $r$ at which for the softest (with maximal $a \sim r$ ) wave-packet the gradient of $\phi$ becomes of order $M_{*}^{2}$.

Indeed, consider a field $\phi_{1}$ produced by self-sourcing of the wave-packet $\phi_{0}$ localized within the sphere of radius $r$. Outside the sphere the field is given by (50), its gradient being of order

$$
\partial_{r} \phi_{1} \sim \frac{A^{3} L_{*}^{4}}{a r^{5}}
$$

\footnotetext{
${ }^{2}$ This can be understood as a consequence of the relations that hold (for $t<0$ ) for any wellbehaved function $Q(x)$,

$$
4 \frac{\delta(r+t)}{r}\left(\frac{d Q(x)}{d x}\right)_{x=-2 r}=\square \frac{\theta(r+t) Q(t-r)}{r}
$$

and

$$
4 \pi Q(t) \delta(\vec{r})=\square \frac{Q(t-r)}{r} .
$$

This shows that an averaged source $Q(t) \delta(\vec{r})$ that gives the same $r>-t$ wave, differs from the exact one by a factor $c=\left.4 t d_{x} Q\right|_{x=2 t} Q(t)^{-1}$. In our case, $Q(x) \propto 1 / x^{3}$ and thus, $c=-3 / 4$.
} 
Equating this to $M_{*}^{2}$, we get,

$$
r \sim L_{*}\left(\frac{A^{3} L_{*}}{a}\right)^{\frac{1}{5}} .
$$

Translating this value in terms of the center of mass energy $\sqrt{s}$, given by (55), we get,

$$
r \sim L_{*}\left(\sqrt{s^{3}} L_{*}^{2} a\right)^{\frac{1}{10}} .
$$

$r_{*}$ is given by the value of $r$ in the above expression, for which the spread $a$ is maximal, that is, $a \sim r$. This gives (56).

The equation (56) confirms the outline of [1] for the scattering process. Since we are not solving the full scattering problem, we can only give qualitative estimates of the outcome, but relation (56) gives us a very important information. This relation indicates, that the size of classical configuration grows with center of mass energy, implying that few-to-few particle scatterings can only go through very low momentum-transfer $\sim M_{*}\left(L_{*} \sqrt{s}\right)^{-\frac{1}{3}}$, and thus have an amplitude $\sim\left(L_{*} \sqrt{s}\right)^{-\frac{4}{3}}$. For $\sqrt{s} \gg M_{*}$, this is a negligible contribution into the scattering cross section, which has to be dominated by many-particle production through the decay of the classical configuration, and can be estimated to have a geometric cross-section, $\sigma \sim L_{*}^{2}\left(L_{*} \sqrt{s}\right)^{\frac{2}{3}}$

\section{$5 \quad$ Importance of Energy-Sourcing}

As in the case of monochromatic waves, the energy-sourcing is a defining property for classicalization of localized wave-packets. We wish to stress, that classicalization is not equivalent to a standard dispersion of a wave-packet in a generic self-interacting theory. For classicalization to take place, it is absolutely essential that the field $\phi$ is (self)sourced by the energy. For other types of self-interactions, the wave packet can still spread at short distances, but because the $r_{*}$-radius diminishes with energy, the system will not classicalize in general. As an example, consider the situation in which we replace the derivative self-coupling in (19) by some other non-derivative self-interaction 3

$$
\mathcal{L}=\frac{1}{2}\left(\partial_{\mu} \phi\right)^{2}-\frac{L_{*}^{n-3}}{(n+1)} \phi^{n+1} .
$$

The equation now becomes,

$$
\square \phi=-L_{*}^{n-3} \phi^{n} .
$$

Applying the same perturbative expansion as before, $\phi=\phi_{0}+\phi_{1}+\ldots$, and taking $\phi_{0}$ in form of the collapsing wave (41), we get the following equation for $\phi_{1}$,

$$
\square \phi_{1}=-A^{n} L_{*}^{n-3} \frac{1}{r^{n}} e^{-n \frac{(r+t)^{2}}{a^{2}}} .
$$

\footnotetext{
${ }^{3}$ We thank Alexander Pritzel and Nico Wintergerst for motivating clarification of this question by their numerical studies.
} 
In the approximation of small $a$, this equation is solved by,

$$
\phi_{1}=-\frac{(-2)^{n-1}}{4(n-2)} \sqrt{\frac{\pi}{n}}\left(A^{n} L_{*}^{n-3} a\right) \frac{\theta(r+t)}{(t-r)^{n-2} r} .
$$

This solution indicates that the system does not classicalize, since the $r_{*}$-radius is always below $L_{*}$. Indeed, the conditions $\phi_{1} \sim \phi_{0}$ and $\phi_{1} \sim M_{*}$ are achieved for

$$
r_{*} \sim L_{*} A^{\frac{n-1}{n-2}}\left(\frac{a}{L_{*}}\right)^{\frac{1}{n-2}}
$$

and

$$
r_{*} \sim L_{*} A^{\frac{n}{n-1}}\left(\frac{a}{L_{*}}\right)^{\frac{1}{n-1}}
$$

respectively. Notice, that for $n=3$, the equation (64) reproduces the result (17) of monochromatic wave-scattering in $\phi^{4}$-theory, if we replace $\omega \rightarrow a^{-1}$ and $L_{*}^{n-3} \rightarrow \lambda$.

Now remembering that for a localized wave-packet $a$ cannot exceed $r$, we conclude that for $A \sim 1$, both conditions give $r_{*} \ll L_{*}$. Thus, the system does not classicalize.

The above consideration illustrates the importance of the energy-sourcing for classicalization, and thus the special role of derivatively-coupled scalars, such as Goldstone bosons.

\section{Classicallization of DGP-Galileon}

Another example we wish to consider is given by the action,

$$
\mathcal{L}=\left(\partial_{\mu} \phi\right)^{2}\left(\frac{1}{2}+\frac{L_{*}^{3}}{4} \square \phi\right)+\phi J .
$$

This theory describes the self-interaction of helicity-zero (Stückelberg) component of the DGP graviton, and can be viewed as the decoupling limit [4] of that theory, in which both the graviton Compton wavelength as well as Planck scale are sent to infinity, whereas the scale $L_{*}$ is kept fixed. We shall treat the above theory in its own right, irrespective of its gravitational origin. The remnant of gravitational nature, however, does persist in form of a residual symmetry under Galilean transformations, under which derivative of the scalar shifts by a constant

$$
\partial_{\mu} \phi \rightarrow \partial_{\mu} \phi+c_{\mu}
$$

Because of this, such scalars are sometimes referred to as Galileons [6].

Because, this theory is known to exhibit $r_{*}$-phenomenon [4, 5, 6, 7, 8, both for static as well as for the time-dependent external sources, in [1] it was identified as an interesting candidate for classicalization. After briefly reviewing the static 
argument, we shall extend the analysis to the scattering process. We shall discover, that in scattering of a spherical wave, classicalization does not happen in the leading order in non-linearities. In the other words, the flux of the $\phi$-gradient can only form after the back-reaction on a linearized wave pulse is taken into the account. The origin of this phenomenon is in the Galilean symmetry.

Before going to scattering case, let us briefly review classicalization of the theory by an external source. Consider a localized source at scale $L$ and energy $\sqrt{s}=$ $1 / L$. At distances $r \gg L$ such a source can be approximated by $4 \pi \delta(\vec{r}) \sqrt{s} L_{*}$. The equation of motion

$$
\square \phi+\frac{L_{*}^{3}}{2}\left[(\square \phi)^{2}-\left(\partial_{\mu} \partial_{\nu} \phi\right)^{2}\right]=J
$$

for a spherically symmetric ansatz has an exact solution [6],

$$
\partial_{r} \phi=\frac{r}{2 L_{*}^{3}}\left(1 \pm \sqrt{1+\frac{4 r_{*}^{3}}{r^{3}}}\right)
$$

where $r_{*} \equiv L_{*}\left(L_{*} \sqrt{s}\right)^{1 / 3}$. Thus, at distances $r \gg r_{*}$ we have $\phi \sim L_{*} \sqrt{s} / r$, whereas for $r \ll r_{*}$ we have $\phi \sim \sqrt{r \sqrt{s}} L_{*}^{-1}$. Thus, $r_{*}$ plays a role similar to the Schwarzschild radius and is the scale at which $\phi$ classicalizes. (An analogous effect in massive gravity was observed by Vainshtein in [9].)

We shall now abandon the external source and consider the situation of dynamical self-sourcing, calculating the correction to the zeroth order collapsing spherical wave-packet of the form (41), following the above-mentioned procedure. It is easy to see that the integrated source at this order is zero. That is,

$$
\left[\frac{L_{*}^{3}}{2} \int d^{3} x \partial_{t}\left(\frac{1}{2} \partial_{t}\left(\partial_{\mu} \phi_{0}\right)^{2}-\partial_{t} \phi_{0} \square \phi_{0}\right)\right]_{\phi_{0}=\psi(r+t) / r}=0,
$$

so that the self-sourcing can only appear after the correction to the linearized wavesolution from non-linearity is taken into account. This result is confirmed by the explicit solution, which we obtain below.

The equation of motion (68) with $J=0$ yields that the first perturbation of a collapsing spherical wave-packet satisfies

$$
\begin{aligned}
\square \phi_{1} & =\frac{L_{*}^{3}}{2}\left(\left(\partial_{\mu} \partial_{\nu} \phi_{0}\right)^{2}\right) \\
& =\frac{L_{*}^{3}}{2}\left(2\left(\partial_{t}^{2} \phi_{0}\right)^{2}-2\left(\partial_{t} \partial_{r} \phi_{0}\right)^{2}-\frac{4}{r} \partial_{t}^{2} \phi_{0} \partial_{r} \phi_{0}+6\left(\frac{\partial_{r} \phi_{0}}{r}\right)^{2}\right) \equiv \bar{j}(r, t),
\end{aligned}
$$

where the zeroth order equation

$$
\square \phi_{0}=\left(\partial_{t}^{2}-\partial_{r}^{2}-\frac{2}{r} \partial_{r}\right) \phi_{0}=0
$$


has been used. Using the explicit form of the zeroth order wave-packet (32), the source $\bar{j}$ rewrites as

$$
\bar{j}(r, t)=L_{*}^{3}\left(\frac{2 \psi^{\prime \prime} \psi}{r^{4}}+\frac{2 \psi^{\prime 2}}{r^{4}}-\frac{6 \psi^{\prime} \psi}{r^{5}}+\frac{3 \psi^{2}}{r^{6}}\right) .
$$

Evaluating the last expression for $\psi$ given by (41), and then taking the $a \rightarrow 0$ limit, it can be recast in the following form

$$
\bar{j}(r, t)=L_{*}^{3} A^{2} \sqrt{\frac{\pi}{2}}\left(\frac{a}{r^{4}} \partial_{r}^{2}-\frac{3 a}{r^{5}} \partial_{r}+\frac{3 a}{r^{6}}\right) \delta(r+t) .
$$

Unlike the previous case, the leading 1/a contribution cancels for the DGP-Galileon. Moreover, solving (71) by the methods given in the appendix, we obtain

$$
\phi_{1}=-L_{*}^{3} A^{2} \sqrt{\frac{\pi}{2}} a \frac{\partial_{t} \delta(r+t)}{(t-r)^{2} r} .
$$

Thus the spread of a DGP-Galileon wave-packet does not happen at this order in non-linearity. This difference as compared to the Goldstone case can be traced to the Galilean symmetry (67) of the Lagrangian (66).

\section{Conclusions}

In this note we have studied dynamics of classicalization phenomenon suggested in [1]. For understanding the fundamental difference between the classicalizing theories and the ones that do not exhibit such an effect, we have generalized the notion of classical $r_{*}$-radius to the latter class of theories. An universal physical meaning of $r_{*}$-radius can be defined as of a shortest distance down to which, in a scattering process, particles propagate freely, without experiencing a significant interaction. By default, the scattering cross section then emerges as a geometric cross section set by $r_{*}$, given by (1). The difference between classicalizing and non-classicalizing theories then can be traced to different behaviors of $r_{*}$-radius with the growing energy. The defining property of classicalizing theories is the growth of $r_{*}$-radius with energy.

As an example, we investigated the system of a derivatively self-coupled scalar, that serves as a prototype for Nambu-Goldstone-Stückelberg field. In order to study classicalization in a simple scattering process, we have prepared an initial collapsing $S$-wave-packet, with high energy but small occupation number. In this way, at the initial stage system can be regarded as being in a quantum state with small number of particles with very high center of mass energy. We then observed that as the wave collapses the classical configuration gradually develops because of the self-sourcing of the Goldstone field by its own energy. Self-sourcing (or sourcing) by energy is a crucial factor for classicalization, which makes it different from a simple dispersion 
of the wave-packet in ordinary interacting theories. Increase of energy inevitably produces a growing self-source of the classicalon field, and leads to the creation of a classical configuration of $r_{*}$-radius which confirms the estimate of [1] for the analogous scattering process.

Classicalon is not created as a result of quantum transition, but is developed gradually because of sourcing by the same energy that makes particles scatter. Because of this fact, production of classicalons, in sharp difference from non-perturbative solitons, is not only unsuppressed, but in fact dominates the scattering process at trans-cutoff energies.

We have performed analogous study for the theory of DGP-Galileon, and discovered that in the leading order in self-sourcing the classicalization does not happen. This suppression can be traced to a high-symmetry of the model.

Our analysis indicates that the nature of (self)sourcing crucially determines the outcome of classicalization, and that Goldstone fields appear as interesting candidates for this phenomenon.

\section{Acknowledgments}

We thank Gian Giudice, Cesar Gomez and Alex Kehagias for valuable on-going discussions on classicalization. We thank Lasha Berezhiani for discussions on classicalization in other systems. The work of G.D. was supported in part by Humboldt Foundation under Alexander von Humboldt Professorship, by European Commission under the ERC advanced grant 226371, by David and Lucile Packard Foundation Fellowship for Science and Engineering, and by the NSF grant PHY-0758032. D.P. is supported by the Mark Leslie Graduate Assistantship at NYU.

\section{Appendix A. Explicit Solution}

In this appendix we give a detailed derivation of classicalization for a localized wavepacket in the self-interacting theory, defined by the lagrangian (19).

We start with the equation of motion for the $\phi$-field

$$
\square \phi=-L_{*}^{4} \partial^{\mu}\left(\partial_{\mu} \phi\left(\partial_{\nu} \phi\right)^{2}\right),
$$

and build up the perturbation series in the small parameter $L_{*}^{4}$

$$
\phi=\phi_{0}+\phi_{1}+\ldots .
$$

The first perturbation $\phi_{1}$ on the solution of the free theory is sourced by the localized source $j(r, t)$, composed of the derivatives of the initial-state wave-packet

$$
\square \phi_{1}=-L_{*}^{4} \partial^{\mu}\left(\partial_{\mu} \phi_{0}\left(\partial_{\nu} \phi_{0}\right)^{2}\right)=j(r, t) .
$$

Using the explicit form of the zeroth-order solution (32), the source can be rewritten in the following way

$$
j(r, t)=-L_{*}^{4}\left(2 \frac{\psi^{2} \psi^{\prime \prime}}{r^{5}}+8 \frac{\psi \psi^{\prime 2}}{r^{5}}-12 \frac{\psi^{2} \psi^{\prime}}{r^{6}}+4 \frac{\psi^{3}}{r^{7}}\right) .
$$


Let us take the function $\psi$ in the form of a gaussian $\psi=A e^{-\frac{(r+t)^{2}}{a^{2}}}$, so that the last expression becomes

$$
j(r, t)=-L_{*}^{4} A^{3}\left(\frac{8}{3 a^{2} r^{5}}-\frac{4}{r^{6}} \partial_{r}+\frac{10}{9 r^{5}} \partial_{r}^{2}+\frac{4}{r^{7}}\right) e^{-\frac{3(r+t)^{2}}{a^{2}}} .
$$

Taking the $a \rightarrow 0$ limit and using the relation

$$
\lim _{a \rightarrow 0} e^{-\frac{3(r+t)^{2}}{a^{2}}}=a \sqrt{\frac{\pi}{3}} \delta(r+t)
$$

the source rewrites as

$$
j(r, t)=-L_{*}^{4} A^{3} \sqrt{\frac{\pi}{3}}\left(\frac{8}{3 a r^{5}}-\frac{4 a}{r^{6}} \partial_{r}+\frac{10 a}{9 r^{5}} \partial_{r}^{2}+\frac{4 a}{r^{7}}\right) \delta(r+t) .
$$

We shall solve the laplace equation for each of the four parts of the right hand side of the last expression viewed as separate sources. The full solution will be the superposition of the four waves

$$
\phi_{1}=\phi_{1}^{(1)}+\phi_{1}^{(2)}+\phi_{1}^{(3)}+\phi_{1}^{(4)}
$$

We start with the equation for $\phi_{1}^{(1)}$, sourced by the first term in (80)

$$
\square \phi_{1}^{(1)}=-L_{*}^{4} A^{3} \sqrt{\frac{\pi}{3}} \frac{8}{3 a} \frac{\delta(r+t)}{r^{5}} .
$$

The last equation can be directly solved via the relation

$$
\square^{-1}\left(\frac{\delta(r+t)}{r^{n+2}}\right)=\frac{(-2)^{n+1}}{4 n} \frac{\theta(r+t)}{(t-r)^{n} r},
$$

which leads to the following expression for $\phi_{1}^{(1)}$,

$$
\phi_{1}^{(1)}=-L_{*}^{4} A^{3} \sqrt{\frac{\pi}{3}} \frac{32}{9 a} \frac{\theta(r+t)}{(t-r)^{3} r} .
$$

The latter expression represents the dominant contribution to the spread of the wave-packet at distances $r \gg a$.

Analogously, the field sourced by the second term in (80), satisfies the following equation

$$
\square \phi_{1}^{(2)}=L_{*}^{4} A^{3} \sqrt{\frac{\pi}{3}} 4 a \frac{\partial_{r} \delta(r+t)}{r^{6}}=L_{*}^{4} A^{3} \sqrt{\frac{\pi}{3}} 4 a \frac{\partial_{t} \delta(r+t)}{r^{6}} .
$$


Defining a new function $f_{1}^{(2)}$ through $\phi_{1}^{(2)} \equiv \partial_{t} f_{1}^{(2)}$, we have

$$
\square f_{1}^{(2)}=L_{*}^{4} A^{3} \sqrt{\frac{\pi}{3}} 4 a \frac{\delta(r+t)}{r^{6}}+g(r)
$$

where $g(r)$ is an integration 'constant', which produces a static configuration for $f_{1}^{(2)}$ and is therefore irrelevant for the expression for $\phi_{1}^{(2)}$, so that we can discard it in what follows. The last equation can now be solved

$$
f_{1}^{(2)}=-L_{*}^{4} A^{3} \sqrt{\frac{\pi}{3}} 8 a \frac{\theta(r+t)}{(t-r)^{4} r} .
$$

Differentiating by time, we obtain the expression for $\phi_{1}^{(2)}$

$$
\phi_{1}^{(2)}=-L_{*}^{4} A^{3} \sqrt{\frac{\pi}{3}}\left(8 a \frac{\delta(r+t)}{(t-r)^{4} r}-32 a \frac{\theta(r+t)}{(t-r)^{5} r}\right) .
$$

For finding $\phi_{1}^{(3)}$, we have to solve the following equation

$$
\square \phi_{1}^{(3)}=-L_{*}^{4} A^{3} \sqrt{\frac{\pi}{3}} \frac{10 a}{9} \frac{\partial_{r}^{2} \delta(r+t)}{r^{5}}=-L_{*}^{4} A^{3} \sqrt{\frac{\pi}{3}} \frac{10 a}{9} \frac{\partial_{t}^{2} \delta(r+t)}{r^{5}} .
$$

Let us again introduce an auxiliary field $\phi_{1}^{(3)} \equiv \partial_{t}^{2} f_{1}^{(3)}$, which satisfies

$$
\square f_{1}^{(3)}=-L_{*}^{4} A^{3} \sqrt{\frac{\pi}{3}} \frac{10 a}{9} \frac{\delta(r+t)}{r^{5}}
$$

and is therefore given by

$$
f_{1}^{(3)}=-L_{*}^{4} A^{3} \sqrt{\frac{\pi}{3}} \frac{40 a}{27} \frac{\theta(r+t)}{(t-r)^{3} r} .
$$

Taking the second time derivative, we obtain

$$
\phi_{1}^{(3)}=-L_{*}^{4} A^{3} \sqrt{\frac{\pi}{3}}\left(\frac{40 a}{27} \frac{\partial_{t} \delta(r+t)}{(t-r)^{3} r}-\frac{80 a}{9} \frac{\delta(r+t)}{(t-r)^{4} r}+\frac{160 a}{9} \frac{\theta(r+t)}{(t-r)^{5} r}\right) .
$$

The fourth contribution $\phi_{1}^{(4)}$ can be directly found from its equation of motion

$$
\phi_{1}^{(4)}=-L_{*}^{4} A^{3} \sqrt{\frac{\pi}{3}} \frac{64 a}{5} \frac{\theta(r+t)}{(t-r)^{5} r} .
$$

Superimposing all the contributions, we obtain the exact expression for the first perturbation on the localized wave-packet

$$
\phi_{1}=-\frac{4}{9} L_{*}^{4} A^{3} \sqrt{\frac{\pi}{3}}\left(\frac{8}{a} \frac{\theta(r+t)}{(t-r)^{3} r}+\frac{10 a}{3} \frac{\partial_{t} \delta(r+t)}{(t-r)^{3} r}-2 a \frac{\delta(r+t)}{(t-r)^{4} r}-\frac{16 a}{5} \frac{\theta(r+t)}{(t-r)^{5} r}\right) .
$$




\section{References}

[1] G. Dvali, G.F. Giudice, C. Gomez and A. Kehagias, arXiv :1010.1415 [hep-ph].

[2] For a study of the suppression of non-perturbative contributions in high-energy particle collisions, see e.g., (and references therein),

F. L. Bezrukov, D. Levkov, C. Rebbi, V. A. Rubakov and P. Tinyakov, Phys. Lett. B 574 (2003) 75 [arXiv:hep-ph/0305300]; Phys. Rev. D 68 (2003) 036005 arXiv:hep-ph/0304180].

C. Rebbi and R. L. . Singleton, Phys. Rev. D 54 (1996) 1020 arXiv:hep-ph/9601260.

[3] G. R. Dvali, G. Gabadadze and M. Porrati, Phys. Lett. B 485, 208 (2000) arXiv:hep-th/0005016].

[4] M.A. Luty, M. Porrati and R, Rattazzi, JHEP 0309 (2003) 029 hep-th/0303116].

[5] G. Dvali, S. Hofmann, J. Khoury, Phys. Rev. D76, 084006 (2007). hep-th/0703027 [HEP-TH]].

[6] A. Nicolis, R. Rattazzi, JHEP 0406 (2004) 059 [hep-th/0404159].

[7] C. Deffayet, G. R. Dvali, G. Gabadadze and A. I. Vainshtein, Phys. Rev. D 65, 044026 (2002) arXiv:hep-th/0106001.

[8] A. Gruzinov, arXiv:astro-ph/0112246.

[9] A.I. Vainshtein, Phys. Lett. B 39 (1972) 393 